\title{
Essential Role of Histidine 84 in Elongation Factor Tu for the Chemical Step of GTP Hydrolysis on the Ribosome
}

\author{
Tina Daviter, Hans-Joachim Wieden and Marina V. Rodnina*
}

Institute of Physical

Biochemistry, University of

Witten/Herdecke, Stockumer

Strasse 10, 58448 Witten

Germany

\begin{abstract}
Elongation factor $\mathrm{Tu}$ (EF-Tu) is a GTP-binding protein that delivers aminoacyl-tRNA to the A site of the ribosome during protein synthesis. The mechanism of GTP hydrolysis in EF-Tu on the ribosome is poorly understood. It is known that mutations of a conserved histidine residue in the switch II region of the factor, His84 in Escherichia coli EF-Tu, impair GTP hydrolysis. However, the partial reaction which is directly affected by mutations of His84 was not identified and the effect on GTP hydrolysis was not quantified. Here, we show that the replacement of His84 with Ala reduces the rate constant of GTP hydrolysis more than $10^{6}$-fold, whereas the preceding steps of ternary complex binding to the ribosome, codon recognition and, most importantly, the GTPase activation step are affected only slightly. These results show that His84 plays a key role in the chemical step of GTP hydrolysis. Rate constants of GTP hydrolysis by wild-type EF-Tu, measured using the slowly hydrolyzable GTP analog, GTP $\gamma$ S, showed no dependence on $\mathrm{pH}$, indicating that His84 does not act as a general base. We propose that the catalytic role of His84 is to stabilize the transition state of GTP hydrolysis by hydrogen bonding to the attacking water molecule or, possibly, the $\gamma$-phosphate group of GTP.
\end{abstract}

(c) 2003 Elsevier Ltd. All rights reserved.

\section{Introduction}

Elongation factor Tu (EF-Tu) catalyzes the binding of aminoacyl-tRNA (aa-tRNA) to the A site of the ribosome. EF-Tu consists of three domains, a Ras-like domain 1 (the $G$ domain) that binds GTP or GDP, and two $\beta$-barrel domains, 2 and 3, which together with domain 1 provide a binding surface for the acceptor domain of aa-tRNA. In its active GTP form, EF-Tu forms a high-affinity complex with aa-tRNA ("ternary complex") and delivers it to the A site of the ribosome. Upon codon-anticodon recognition, GTP is hydrolyzed, resulting in a major conformational change of EF-Tu. This triggers the release of the $3^{\prime}$ end of aa-tRNA from EF-Tu and the subsequent dissociation of EF-Tu.GDP from the ribosome. The recycling of the inactive

Abbreviations used: EF-Tu, elongation factor $\mathrm{Tu}$; GTP $\gamma S$, guanosine $5^{\prime}$-[ $\gamma$-thio]triphosphate; mant-GTP,

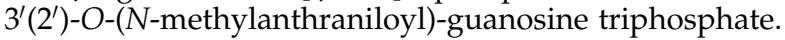

E-mail address of the corresponding author: rodnina@uni-wh.de
GDP-bound form of EF-Tu to EF-Tu.GTP is catalyzed by elongation factor Ts (EF-Ts).

GTP hydrolysis is central to the function of EF-Tu, but its mechanism is not understood. By analogy to other GTP-binding proteins, the reaction is likely to proceed by a direct in-line attack of a water molecule on the $\gamma$-phosphorus atom of GTP. ${ }^{1,2}$ The catalytic residues involved in the reaction in EF-Tu and their role in catalysis have not been identified. Early work suggested that the conserved Gln residue present in the switch II region of all Ras-like and heterotrimeric $G$ proteins could act as a general base by abstracting a proton from the water. $^{1,2}$ However, both theoretical ${ }^{3,4}$ and experimental ${ }^{5,6}$ analysis showed that the $\mathrm{p} K_{\mathrm{a}}$ of the Gln in question is too low for a general-base function. Rather, the $\gamma$-phosphate group itself appears to act as a base (substrate-assisted catalysis ${ }^{5}$ ). Crystal structures of several Ras-like and heterotrimeric G proteins indicated that the Gln residue in the switch II region is crucial for the stabilization of the GTPase transition state by correct positioning of both the water molecule and the $\gamma$-phosphate, 
providing a likely explanation for the strong inhibitory effects of replacing the conserved Gln with other amino acid residues. ${ }^{7}$

In all members of the translation factors subfamily of GTPases, ${ }^{8}$ the position of the conserved Gln in the switch II region is occupied by His, His 84 in EF-Tu from Escherichia coli. His84 could act in a similar way as Gln in stabilizing the GTPase transition state. However, in the structures of EF-Tu in the GTP form, His84 points away from the catalytic site and its access to the $\gamma$-phosphate group and the hydrolytic water molecule is prohibited by the side-chains of Val20 and Ile60 that form a hydrophobic gate. ${ }^{2}$ It was suggested that, in order to accelerate GTP hydrolysis, one or both of the wings of the hydrophobic gate could open, providing access of His84 to the $\gamma$-phosphate. ${ }^{9}$ The switch II region that comprises His84 undergoes a major reorientation during the transition from the GTP to the GDP form, or upon interaction of EF-Tu with EF-Ts. Thus, a reorientation of His84 during GTPase activation is structurally possible. In fact, in the structure of EF-Tu.GDP in the complex with aurodox, an antibiotic which stimulates the GTPase activity of EF-Tu, the position of Ile60 is disordered and His84 is oriented towards the $\gamma$-phosphate position within the nucleotide-binding site. ${ }^{10}$ However, because the stimulation of GTP hydrolysis by aurodox is only tenfold (albeit measured under conditions of multiple turnover), ${ }^{11}$ compared to the $10^{5}$-fold activation on the ribosome, ${ }^{12}$ and the activation by the antibiotic and the ribosome may proceed by different mechanisms, ${ }^{13}$ the functional significance of these structural data remained unclear. ${ }^{10}$

In the isolated $\mathrm{G}$ domain of EF-Tu, the exchange of His84 for Gly led to a moderate (20-fold) reduction of the intrinsic GTPase activity measured in the absence of the ribosomes, much less than expected for the removal of a residue directly involved in catalysis. ${ }^{14}$ Substitution of His84 in full-size EF-Tu from E. coli reduced (H84Q) or abolished (H84A) the GTPase activity of EF-Tu alone or bound to non-programmed ribosomes. ${ }^{15}$ Similar results were obtained for EF-Tu from Thermus thermophilus when the H85Q and H85L substitutions at the position homologous to H84 in E. coli EF-Tu were studied, whereas the H85G mutation caused complete protein degradation in vivo. ${ }^{16}$ Upon ternary complex binding to poly(U)programmed ribosomes, the rate of GTP hydrolysis was moderately reduced by the His substitution to Gln in EF-Tu from E. coli (no quantitative estimation of inhibition) or T.thermophilus (12-fold inhibition). These results suggest that Gln may take over part, but not all of the functions of the presumed catalytic His in the switch II region. The H85L substitution in T. thermophilus EF-Tu abolished the GTPase activity on poly(U)-programmed ribosomes. ${ }^{16}$ The activity of the H84A mutant of E. coli EF-Tu on mRNA-programmed ribosomes was not analyzed. ${ }^{15}$

These studies suggested an important role of
His84 in the catalysis of GTP hydrolysis in EF-Tu on the ribosome. However, several important questions remained unresolved. First, GTP hydrolysis on the ribosome follows, and is rate-limited by, the preceding steps, including binding of the ternary complex to the ribosome, codon-anticodon interaction, and a conformational change of the factor leading to GTPase activation. Mutation of His84 could affect any of these preceding steps, rather than the chemistry step itself, resulting in an apparent effect on GTP hydrolysis. This issue has not been addressed in previous work. Second, the quantitative contribution of His84 to catalysis of GTP hydrolysis by ribosome-bound EF-Tu was not determined. Finally, unlike Gln, the side-chain of His has a $\mathrm{pK}_{\mathrm{a}}$ at neutral $\mathrm{pH}$ and may act as a general base, in addition to the substrate-assisted catalysis, a possibility which has not been examined previously. Here, we analyze the role of His 84 in catalysis of GTP hydrolysis in EF-Tu on poly(U)-programmed ribosomes. We have studied the H84A mutant of EF-Tu in order to identify the precise point within the functional cycle of EF-Tu where His84 may exert its function. Kinetic analysis was carried out to quantify the contribution of His to overall catalysis of GTP hydrolysis. Finally, in order to determine whether His84 may function in general acid-base catalysis, the $\mathrm{pH}$ dependence of GTP cleavage of the slowly hydrolyzable GTP analog, GTP $\gamma S$, was studied in the $\mathrm{pH}$ range between 6.5 and 8.5 .

\section{Results}

\section{Kinetics of GTP hydrolysis and peptide bond formation}

The H84A mutant of EF-Tu was prepared and purified as described in Materials and Methods. Nucleotide and aa-tRNA binding were not affected by the mutation (data not shown), in agreement with previous reports for EF-Tu from E. coli ${ }^{15}$ and T. thermophilus. ${ }^{16}$ The ternary complex, EF$\mathrm{Tu}(\mathrm{H} 84 \mathrm{~A}) \cdot \mathrm{GTP} \cdot \mathrm{Ph}_{\mathrm{e}}-\mathrm{tRNA}{ }^{\text {Phe }}$, was stable enough to allow its purification from unbound ligands by gel-filtration as described. ${ }^{17}$

The rate of GTP hydrolysis was measured upon addition of the ternary complex, EF$\mathrm{Tu}(\mathrm{H} 84 \mathrm{~A}) \cdot \mathrm{GTP} \cdot \mathrm{Phe}$-tRNA ${ }^{\text {Phe }}$, to poly(U)-programmed ribosomes carrying an analog of peptidyl-tRNA, AcPhe-tRNA ${ }^{\text {Phe }}$, in the $\mathrm{P}$ site (referred to as $\mathrm{P}$ siteblocked ribosomes) (Figure 1). GTP hydrolysis was very slow, with a rate constant $k_{\mathrm{GTP}}=$ $3.0( \pm 0.3) \times 10^{-4} \mathrm{~s}^{-1}$. Under the same conditions, GTP hydrolysis with wild-type EF-Tu is very rapid $\left(k_{\mathrm{GTP}}>500 \mathrm{~s}^{-1}\right.$ with both wild-type EF-Tu and EF-Tu with an oligohistidine tag as the H84A mutant studied here ${ }^{12,18}$ ) and its actual rate is limited by the slowest of the preceding steps, i.e. codon recognition. On non-programmed ribosomes, GTP hydrolysis was extremely slow, $3 \times 10^{-5} \mathrm{~s}^{-1}$, suggesting that GTP hydrolysis by 
mutant EF-Tu is stimulated tenfold by a correct codon-anticodon interaction. In addition, peptide bond formation was very slow with EF-Tu(H84A), $k_{\text {pep }}=2.6( \pm 0.2) \times 10^{-4} \mathrm{~s}^{-1}$. With wild-type EF-Tu, the rate of peptide bond formation reached $8 \mathrm{~s}^{-1}$ and was limited by the accommodation of aatRNA in the A site. ${ }^{12,18}$ The fact that $k_{\mathrm{GTP}}$ and $k_{\text {pep }}$ were the same with mutant EF-Tu indicates that peptide bond formation was limited by the rate of GTP hydrolysis.

\section{Affinity of ribosome binding}

The low rate of GTP hydrolysis may result from either inefficient catalysis or weak binding of the mutant ternary complex to the ribosome. To distinguish between these two possibilities, we tested the stability of ternary complex binding to the ribosome. The ribosome complex with EF$\mathrm{Tu}(\mathrm{H} 84 \mathrm{~A}) \cdot\left[{ }^{3} \mathrm{H}\right] \mathrm{GTP} \cdot\left[{ }^{14} \mathrm{C}\right]$ Phe-tRNA ${ }^{\text {Phe }}$ was formed using a slight excess of $\mathrm{P}$ site-blocked ribosomes, and isolated from unbound ligands by gel-filtration on Sephacryl S300. The elution position of ternary complex was identified by immunoblotting using antibodies against the histidine tag of EF-Tu and by counting ${ }^{3} \mathrm{H}$ and ${ }^{14} \mathrm{C}$ radioactivity. Because of the large difference in the molecular mass between the ribosomes and the free ternary complex, a clear separation of the respective peaks was found, and in the presence of ribosomes practically the whole amount of ternary complex eluted in the ribosome peak (data not shown). The complex was stable and could be isolated and quantified by nitrocellulose filtration. Upon longer incubations (15-60 minutes), the amount of $\left[{ }^{3} \mathrm{H}\right] \mathrm{GTP}$ bound to the complex decreased, due to GTP hydrolysis (Figure 1) and dissociation of EF-Tu. $\left[{ }^{3} \mathrm{H}\right] \mathrm{GDP}$ from the ribosome (EF-Tu. $\left[{ }^{3} \mathrm{H}\right] \mathrm{GDP}$ was poorly retained on the nitrocellulose filters under the filtration conditions used).

The reversibility of mutant ternary complex

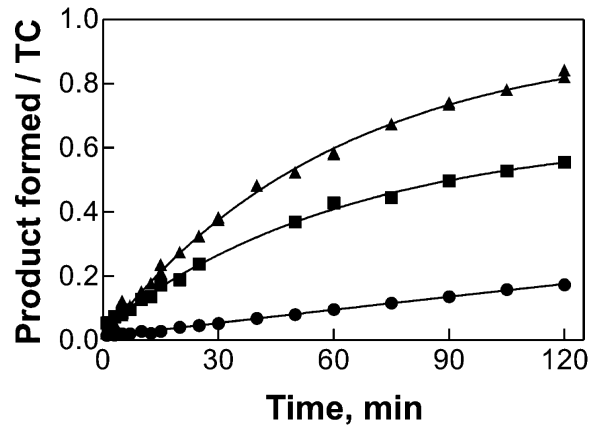

Figure 1. Functional activity of EF-Tu(H84A). Peptide bond formation (squares) and GTP hydrolysis were measured upon interaction of EF-Tu(H84A). $[\gamma-$ $\left.{ }^{32} \mathrm{P}\right] \mathrm{GTP} \cdot\left[{ }^{14} \mathrm{C}\right] \mathrm{Phe}$-tRNA ${ }^{\text {Phe }}(0.1 \mu \mathrm{M})$ with $\mathrm{P}$ site-blocked ribosomes $(0.3 \mu \mathrm{M})$ (triangles) or non-programmed ribosomes $(0.3 \mu \mathrm{M})$ (circles). Plotted is the extent of product formation relative to the amount of ternary complex (TC) added.

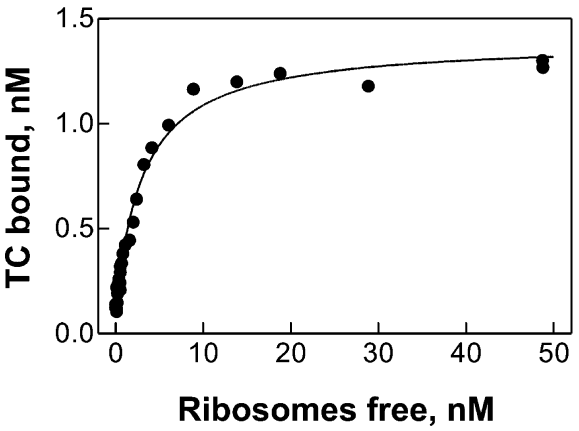

Figure 2. Affinity of EF-Tu(H84A).GTP. $\left[{ }^{14} \mathrm{C}\right] \mathrm{Phe}-$ tRNA $^{\text {Phe }}$ to the ribosome complexes. Ternary complex (TC) binding to $\mathrm{P}$ site-blocked ribosomes was determined by nitrocellulose filtration one minute after mixing (Materials and Methods). For details, see the text.

binding to the ribosome was tested by chase experiments. The formation of the complex was complete within one minute after mixing. Upon short incubations (several minutes), when GTP was not hydrolyzed significantly, binding of EF-Tu(H84A).GTP. $\left[{ }^{14} \mathrm{C}\right]$ Phe-tRNA ${ }^{\text {Phe }}$ to the ribosome was reversible, because the addition of excess unlabeled ternary complex resulted in rapid dissociation of the mutant ternary complex from the ribosome (data not shown). The equilibrium binding constant of mutant ternary complex, EFTu(H84A).GTP.Phe-tRNA ${ }^{\text {Phe }}$, to the ribosome was determined by titrating a constant amount of ternary complex with increasing amounts of ribosomes and determining complex formation by nitrocellulose filtration (Figure 2). The $K_{\mathrm{d}}$ of the complex was estimated to $3.1 \pm 0.3 \mathrm{nM}$, consistent with the fact that the complex could be isolated by gelfiltration, as described above. In comparison, the $K_{\mathrm{d}}$ of wild-type ternary complex binding to the ribosome was about $0.5 \mathrm{nM}$, as calculated from the rate constants of the steps preceding GTP hydrolysis. ${ }^{12,18}$ Thus, the affinity of ternary complex binding to the ribosome is not much affected by the H84A mutation.

\section{Kinetic analysis of A-site binding}

To identify the step in the mechanism of A-site binding which is impaired by the mutation and to quantify the effects on this and all other steps, a kinetic analysis was performed, as described previously. ${ }^{12}$ The rate constants of initial binding of the ternary complex to the ribosome and subsequent codon recognition were determined using a fluorescent RNA $^{\text {Phe }}$ derivative, PhetRNA $^{\text {Phe }}\left(\right.$ Prf16/17). ${ }^{17}$ Initial binding of ternary complex to the ribosome was measured by monitoring the fluorescence change of EFTu(H84A).GTP.Phe-tRNA ${ }^{\text {Phe }}(\operatorname{Prf16} / 17)$ upon mixing with non-programmed ribosomes, which is equivalent to initial complex formation with programmed ribosomes. ${ }^{12,19}$ Apparent rate constants 
were determined from time-courses measured at different ribosome concentrations by exponential fitting and plotted versus the ribosome concentration (Figure 3(a)). From the slope of the linear plot, the bimolecular association rate constant was calculated, $k_{1}=120( \pm 20) \mu \mathrm{M}^{-1} \mathrm{~s}^{-1}$. From the intercept with the $Y$-axis, the dissociation rate constant of the initial-binding complex, $k_{-1}=75( \pm 25) \mathrm{s}^{-1}$, was determined. The $K_{\mathrm{d}}$ of initial binding calculated from these values $\left(K_{\mathrm{d}}=k_{-1} / k_{1}=0.63 \mu \mathrm{M}\right)$ was the same as that determined from the analysis of the concentration dependence of the fluorescence amplitudes (not shown), $0.6 \mu \mathrm{M}$, and was similar to the value found for ternary complex containing wild-type EF-Tu. ${ }^{12,19}$

When the ternary complex, EF-Tu(H84A).GTP. Phe-tRNA $^{\text {Phe }}(\operatorname{Prf16} / 17)$, was mixed with poly(U)programmed ribosomes, an increase in fluorescence was observed within the first second (Figure 3(b)), followed by a slow decrease in fluorescence (not shown). Exponential fitting yielded apparent rate constants of about $10-40 \mathrm{~s}^{-1}$ and $6 \mathrm{~s}^{-1}$ describing the increase in fluorescence. The decrease in fluorescence had an apparent rate of about $0.5 \mathrm{~s}^{-1}$. The rate of the fastest step increased with ribosome concentration in a hyperbolic fashion (Figure 3(c)), consistent with a monomolecular rearrangement step following a bimolecular association reaction. The same experiments were performed monitoring the fluorescence of wybutine, a natural fluorophore at position 37 of $\mathrm{tRNA}^{\text {Phe }}$, which specifically reports the formation of the codon-anticodon complex. ${ }^{20,21}$ This yielded the same rates as the fastest step seen with proflavin (Figure 3(c)), indicating that the step in question was codon recognition. Since the codon recognition complex with cognate aa-tRNA is rather stable, i.e. $k_{-2}$ is small compared to $k_{2}{ }^{12,22}$ the value of the rate constant of codon recognition can be estimated from the saturation of a hyperbolic fit, $k_{2}=50( \pm 10) \mathrm{s}^{-1}$. The apparent $K_{\mathrm{M}}$ of the codon-recognition step calculated from the ribosome concentration at half-saturation was $0.7( \pm 0.2) \mu \mathrm{M}$, in agreement with the $K_{\mathrm{M}}$ value calculated from the kinetic constants, $\left(k_{-1}+k_{2}\right) /$ $k_{1}=1.0( \pm 0.4) \mu \mathrm{M}$. The nature of the slow fluorescence changes is not known. The rates of these reactions were independent of the ribosome concentration, about $6 \mathrm{~s}^{-1}$ and $0.5 \mathrm{~s}^{-1}$ at saturation, (a)

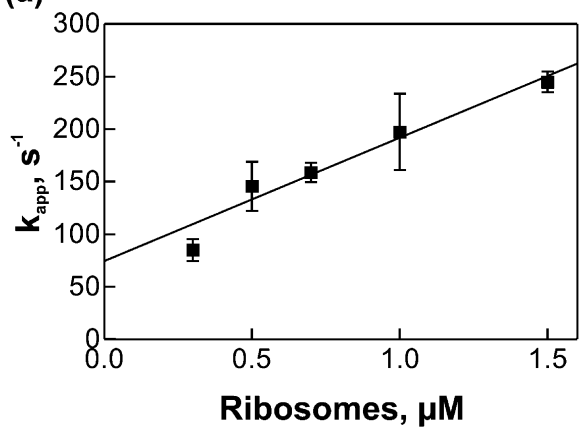

(c)

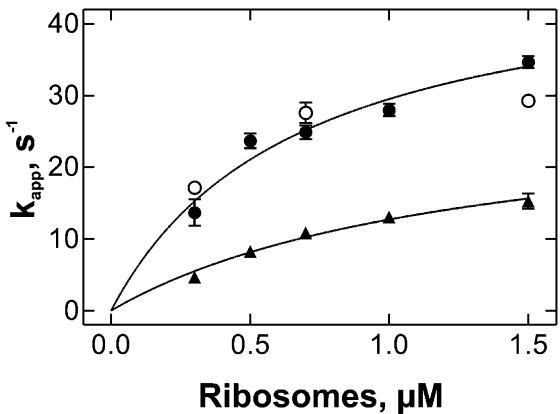

(b)

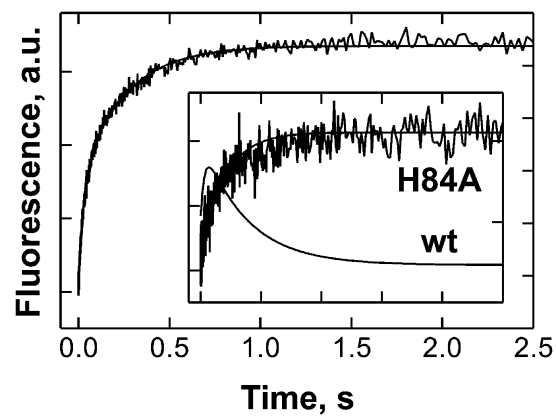

(d)

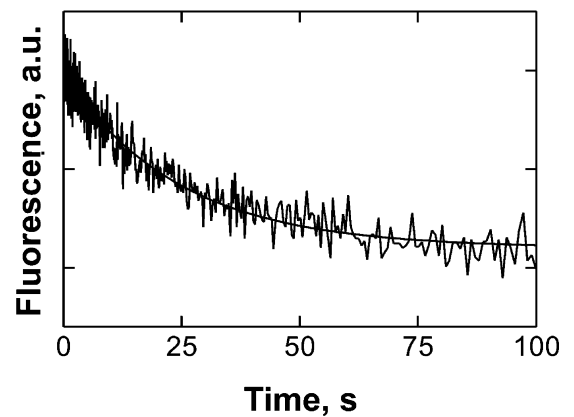

Figure 3. Kinetic analysis of A-site binding. (a) Initial binding. The apparent rate constants were determined by exponential fitting of the time-courses of the fluorescence changes measured after mixing of the purified ternary complexes EF-Tu(H84A).GTP.Phe-tRNA ${ }^{\text {Phe }}($ Prf16/17) $(0.1 \mu \mathrm{M})$ with increasing amounts of non-programmed ribosomes. (b) Codon recognition and GTPase activation. Fluorescence changes of proflavin in $\mathrm{tRNA}^{\text {Phe }}($ Prf16/17) were measured after mixing of ternary complex containing EF-Tu(H84A) $(0.1 \mu \mathrm{M})$ with $\mathrm{P}$ site-blocked ribosomes $(0.3 \mu \mathrm{M})$ in the stopped-flow apparatus. Inset, analogous experiment monitoring mant-GTP fluorescence; time-scale as in the main Figure. The fit of the analogous curve obtained the wt EF-Tu is shown for comparison. (c) Concentration dependence of $k_{\text {app2 }}$ (codon recognition) and $k_{\text {app3 }}$ (GTPase activation) monitored by the fluorescence of proflavin (filled circles), wybutine (open circles), or mant-GTP (triangles). (d) Dissociation from the ribosome $\left(k_{-2}\right)$. The dissociation of EF-Tu(H84A).GTP.Phe-tRNA ${ }^{\text {Phe }}($ Prf16/17) from the ribosome was initiated by the addition of unlabeled ternary complex in tenfold excess and proflavin fluorescence was monitored. 
and were much faster than GTP hydrolysis with mutant EF-Tu. Hence, the fluorescence changes may reflect rearrangements of the complex upon or after GTPase activation but before GTP hydrolysis. Because this step is not observable with wild-type EF-Tu due to rapid GTP hydrolysis and its structural and functional meaning is unclear, it was not included in the kinetic model.

The kinetic stability of the codon recognition complex, $k_{-}$, was determined by chase experiments (Figure 3(d)). The dissociation of EF-Tu(H84A). GTP.Phe-tRNA ${ }^{\text {Phe }}(\operatorname{Prf16} / 17)$ from the ribosome was initiated by adding unlabeled ternary complex in large excess. Under these conditions, the dissociation rate represents $k_{-2}$. With cognate PhetRNA $^{\text {Phe }}, k_{-2}=0.04( \pm 0.01) \mathrm{s}^{-1}$ was obtained by exponential fitting of the time-courses as in Figure 3(d). As expected, $k_{-2}$ increased significantly, to about $2 \mathrm{~s}^{-1}$, when near-cognate Leu-tRNA ${ }^{\text {Leu2 }}$ (anticodon GAG) was used (not shown), because the stability of the codon-recognition complex strongly depends on the codon-anticodon match. 22,23

The crucial step for GTP hydrolysis is GTPase activation, i.e. the ribosome-induced rearrangement of EF-Tu that leads to the GTPase transition state and subsequent GTP hydrolysis. The GTPase activation rearrangement was monitored by the fluorescence of a derivative of GTP, mantGTP. ${ }^{12,20,24}$ With wild-type EF-Tu, the interaction of the ternary complex with the ribosome led to a biphasic change in mant fluorescence, with an increase in fluorescence reflecting GTPase activation, and a decrease in fluorescence due to dissociation of EF-Tu-mant-GDP from the ribosome after GTP hydrolysis. ${ }^{12,18,20}$ Upon mixing of EF$\mathrm{Tu}(\mathrm{H} 84 \mathrm{~A}) \cdot$ mant-GTP.Phe-tRNA $^{\text {Phe }}$ with P siteblocked ribosomes, an increase in mant fluorescence was observed similar to that found with wild-type EF-Tu, while no decrease in fluorescence was observed within the time of the stopped-flow experiment (Figure 3(b), inset). This indicates that the mutation does not affect the GTPase-activation rearrangement, but rather inhibits subsequent steps. The analysis of the concentration dependence of the rates showed a hyperbolic behavior with saturation at $k_{\text {app }}=30( \pm 5) \mathrm{s}^{-1}$ (Figure 3(c)). For the kinetic mechanism of A-site binding depicted in Figure 5, this value is equal to $k_{2} k_{3}$ / $\left(k_{2}+k_{3}\right) \cdot{ }^{25}$. Using the value of $k_{2}=50( \pm 10) \mathrm{s}^{-1}$, determined above, the rate constant of the GTPase activation was calculated to $k_{3}=70( \pm 20) \mathrm{s}^{-1}$. The summary of rate constants of A-site binding with $\mathrm{EF}-\mathrm{Tu}(\mathrm{H} 84 \mathrm{~A})$ is shown in Figure 4.

The rate constant of the breakdown of the GTPase-activated state, $k_{-3}$, cannot be determined directly from kinetic data. Comparing the overall $K_{d}$, which may be calculated from the rate constant of Figure 4 (Materials and Methods), with that measured experimentally (Figure 2) suggests that the internal equilibrium $k_{-3} / k_{3}=6$, and thus $k_{-3}=420 \mathrm{~s}^{-1}$, which is too fast to be measured directly in chase experiments. However, given the standard deviations of the values of the rate constants and the different experimental approaches used for the determination of equilibrium and rate constants, the value of $k_{-3}$ should be considered as an estimate, and it is therefore not included in Figure 4.

In summary, the H84A mutation reduces the rate of GTP cleavage by more than six orders of magnitude, while the rate constants of other elemental steps of A-site binding, including GTPase activation, are little affected. The small effects on the rate constants $k_{-1}, k_{2}, k_{-2}$, and $k_{3}$ may be explained by a slightly different conformation of the ternary complex containing mutant EF-Tu, compared to the wild-type factor.

\section{pH dependence of GTP hydrolysis}

As His84 in EF-Tu plays a crucial role in the catalysis of GTP hydrolysis, we next asked the question whether it could act as a general base in abstracting a proton from the water molecule during the approach to the transition state of hydrolysis. If a general base were involved in catalysis, the rate of the reaction would be expected to depend on $\mathrm{pH}$ according to the $\mathrm{p} K_{\mathrm{a}}$ of the catalytic base. For histidine, the $\mathrm{p} K_{\mathrm{a}}$ is expected to be near 7 . Thus, the experiment to perform would be to determine the rate constants of GTP hydrolysis by wild-type EF-Tu on the ribosome at different $\mathrm{pH}$ values. However, this experiment is not feasible, because GTP hydrolysis by wild-type EF-Tu is rapid intrinsically and its actual rate is limited by the preceding steps; thus, $k_{\mathrm{GTP}}$ cannot be determined in such an experiment. Therefore, we have used a slowly hydrolyzable derivative of GTP, GTP $\gamma$ S, which is known to be a suitable analog to study the function of EF-Tu on the ribosome ${ }^{26}$ following the same mechanism with respect to substrate-assisted catalysis as GTP, at least in Ras. ${ }^{5}$ Furthermore, in order to control whether GTPase activation is also affected by $\mathrm{pH}$, we used a fluorescent derivative of GTP $\gamma S$, mant-GTP $\gamma S$. Upon interaction of EF-Tu-mant-GTP $\gamma S$.Phe-tRNA ${ }^{\text {Phe }}$ with $\mathrm{P}$ site-blocked ribosomes at $\mathrm{pH} 7.5$, an increase of mant fluorescence was observed, followed by a very slow decrease (Figure 5(a)). As determined with mant-GTP, the increase in fluorescence reports the GTPase activation rearrangement of EF-Tu. ${ }^{20}$ The rate of this step as reported by mant-GTP $\gamma S, 7.2 \pm 0.8 \mathrm{~s}^{-1}$, is somewhat slower than that obtained with mant-GTP at the same ribosome concentration, $18 \mathrm{~s}^{-1,12,18}$ but is still within the same kinetic domain, indicating that the modification of the $\gamma$-phosphate of mantGTP $\gamma S$ had little effect on the steps of A-site binding up to and including the GTPase activation. However, the rate of the slow step is decreased about 400 -fold, from about $3 \mathrm{~s}^{-1}$ to $0.008 \mathrm{~s}^{-1}$, upon replacement of mant-GTP by mant-GTP $\gamma \mathrm{S}$, consistent with very slow hydrolysis of GTP $\gamma S .{ }^{26}$ This suggests that the slow decrease in mant fluorescence is determined by the rate of the preceding 

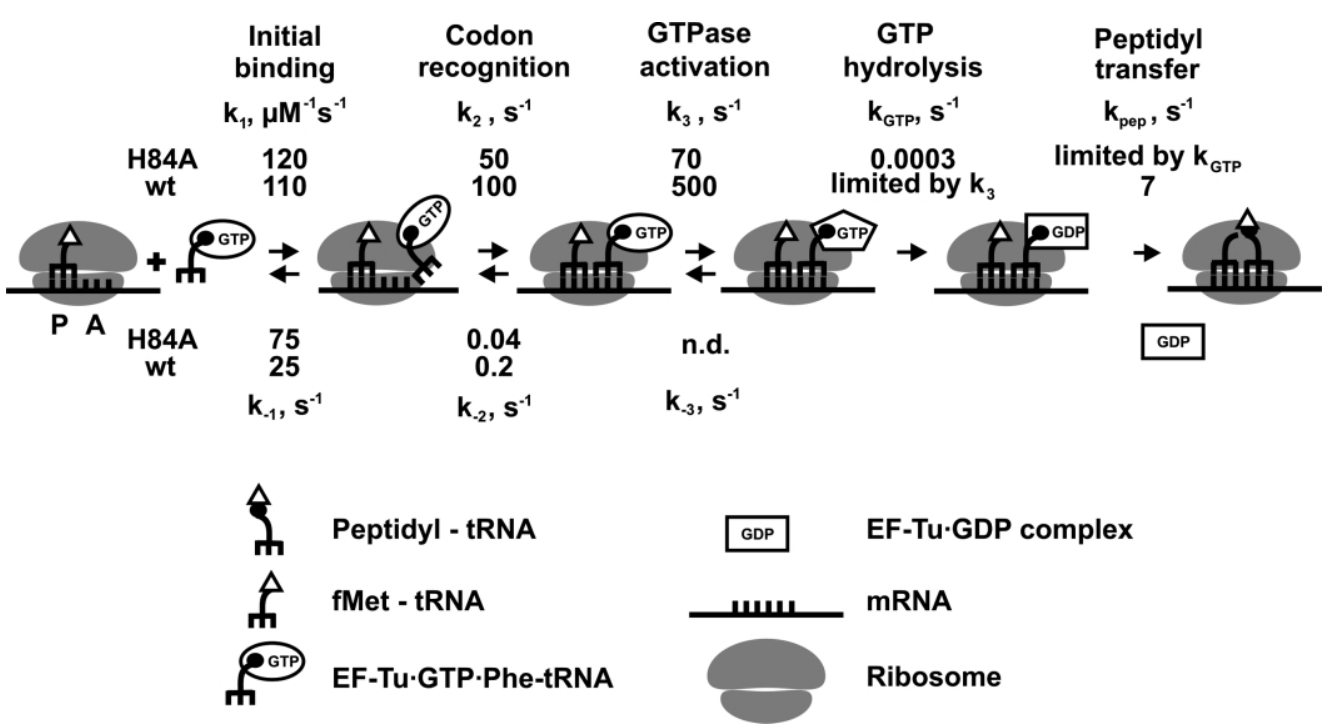

Figure 4. Kinetic scheme of A-site binding with EF-Tu(H84A). The scheme summarizes the rate constants determined by kinetic analysis, as described in the text. The rate constants for wild-type EF-Tu ${ }^{12}$ are shown for comparison.

(a)

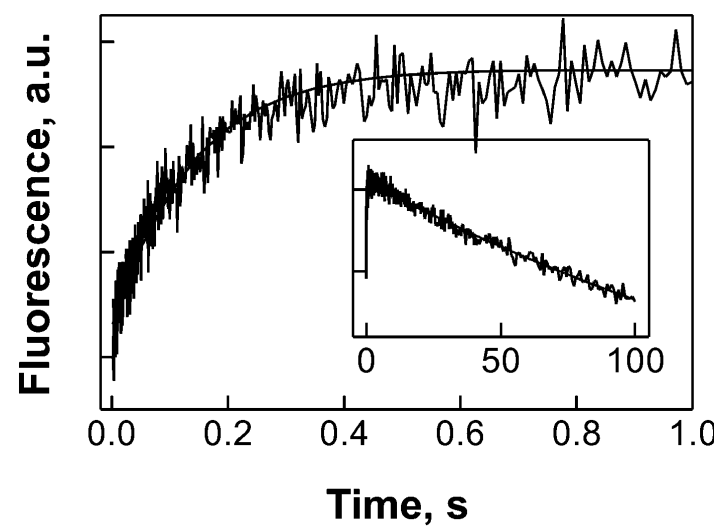

(b)

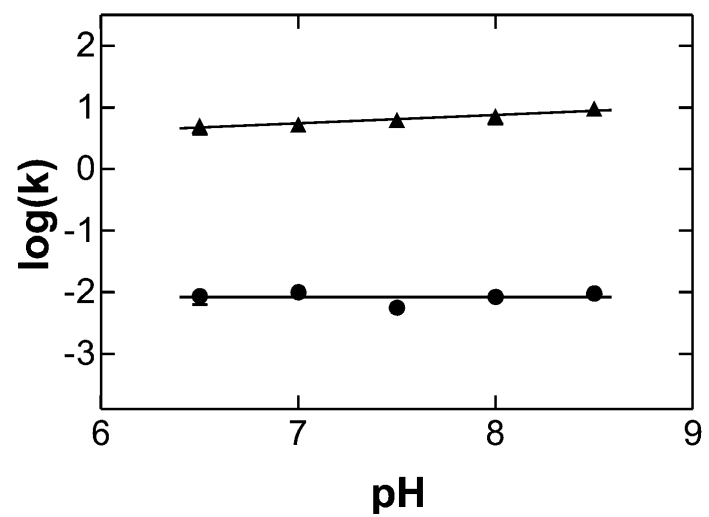

Figure 5. $\mathrm{pH}$ dependence of GTPase activation and GTP hydrolysis. (a) Time-course of GTPase activation (rapid fluorescence increase) and GTP hydrolysis (slow decrease in fluorescence, inset) measured with wildtype EF-Tu with a slowly hydrolyzable fluorescent GTP analog, mant-GTP $\gamma \mathrm{S}$, at $\mathrm{pH}$ 7.5. Main Figure, short timewindow; inset, long-time window of the same experiment. (b) $\mathrm{pH}$ dependence of the rates of GTPase activation (triangles) and GTP hydrolysis (circles). hydrolysis of mant-GTP $\gamma \mathrm{S}$. Thus, the rate of the slow step provides a measure for $k_{\mathrm{GTP} \gamma \mathrm{S}}$ and can be monitored to determine the $\mathrm{pH}$ dependence of the reaction.

Experiments analogous to those described above at $\mathrm{pH} 7.5$ were carried out at $\mathrm{pH} 6.5$ to 8.5 . A broader range of $\mathrm{pH}$ values could not be tested, because of EF-Tu aggregation at $\mathrm{pH}$ values below $6.5^{27}$ (T.D., H.-J.W. \& M.V.R., unpublished results) and significant hydrolysis of Phe-tRNA ${ }^{\text {Phe }}$ above $\mathrm{pH} 8.5$, both rendering the ternary complex inactive. The rate constants of GTPase activation and GTP $\gamma S$ hydrolysis showed no dependence on $\mathrm{pH}$ (Figure 5(b)). This is at variance with the expected behavior if a general base with a $\mathrm{p} K_{\mathrm{a}}$ of about 7 were involved in catalysis. These results strongly argue against the involvement of a general base ionizing at neutral $\mathrm{pH}$ in the catalytic mechanism of GTP hydrolysis by EF-Tu on the ribosome.

\section{Discussion}

His84 in the switch II region of EF-Tu plays a key role in the mechanism of GTP hydrolysis on the ribosome. The present kinetic results show that the side-chain of His 84 is directly involved in the chemistry step of the reaction, while all the steps preceding GTP cleavage are practically not affected by the H84A mutation. Notably, the GTPase activation rearrangement of domain 1 of EF-Tu, which can be envisaged as a conformational change leading to opening of the hydrophobic gate, ${ }^{2,9}$ is not inhibited by the mutation. The replacement of His 84 by Ala decreased the rate of GTP hydrolysis by more than six orders of magnitude, which translates into an increase in the activation energy barrier by $35 \mathrm{~kJ} / \mathrm{mol}$. EF-Tu with an H84Q substitution hydrolyzed GTP with rates that were somewhat lower, but comparable to those of wild-type 
EF-Tu. ${ }^{15,16}$ Furthermore, the present data on the $\mathrm{pH}$ dependence of the rate of GTP hydrolysis disfavors a general-base function of His84 in the reaction. Thus, the likely catalytic role of His 84 is to form hydrogen bond(s) to the substrates of the reaction, the attacking water molecule and/or the $\gamma$-phosphate, to precisely align groups directly involved in the reaction, in a similar way as Gln stabilizes the transition state of GTP hydrolysis in Ras-like or heterotrimeric $\mathrm{G}$ proteins ${ }^{7}$ (Figure 6).

Spontaneous GTP hydrolysis in EF-Tu is very slow, about $5 \times 10^{-5} \mathrm{~s}^{-1} .{ }^{28}$ In the structure of EFTu.GTP, His84 points away from the $\gamma$-phosphate, and its rotation towards the active site is hindered by Ile60 and Val20 (Figure 6). The conformation of
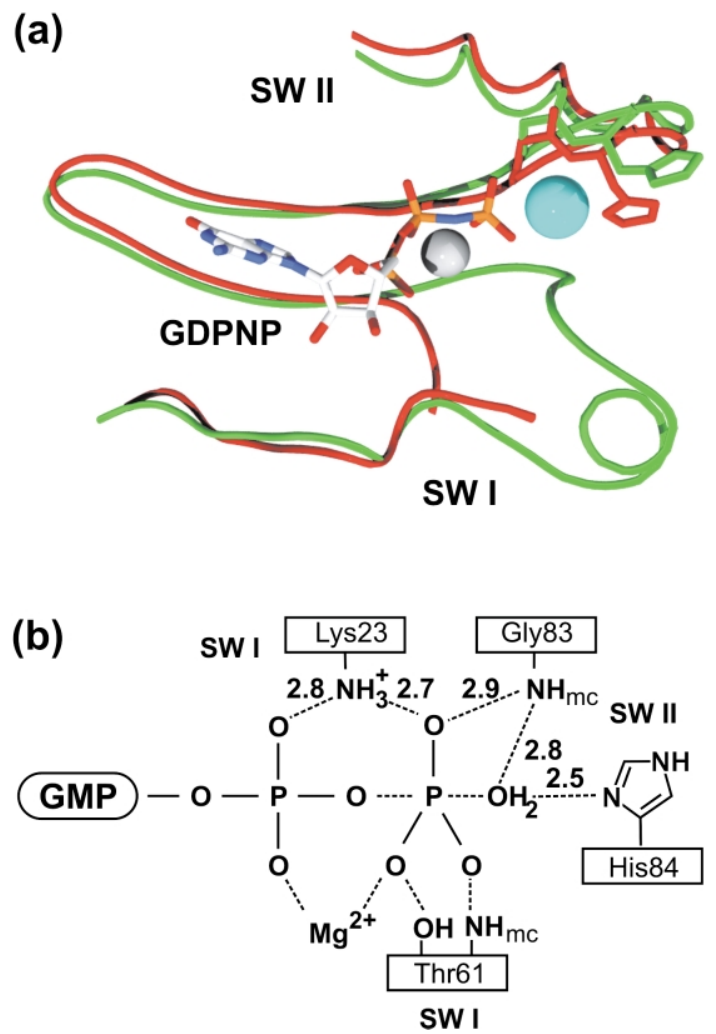

Figure 6. Orientation of His84 in the active site of EFTu. (a) Positions of His84 in the crystal structures of EFTu.GDPNP (green, PDB 1EXM) and EF-Tu.GDP.aurodox (red, 1HA3), are shown relative to the hydrolytic water molecule (cyan), $\mathrm{Mg}^{2+}$ (gray) and GDPNP (1EXM). Structures were aligned on Lys23 of EF-Tu which occupies the same position in the different structures. SWI and SWII, switch I and II regions. Part of the switch I region (amino acid residues 53-61) is not resolved in the EF-Tu.GDP.aurodox structure. (b) Model of the transition state of GTP hydrolysis. Interactions between residues important for catalysis and GTP are inferred from the superposition of the EF-Tu.GDPNP and the EF-Tu.GDP.aurodox structures. The positions of Lys23, Gly83 and His84 are taken from the EF-Tu.GDP.aurodox complex (1HA3), those of water, $\mathrm{Mg}^{2+}, \mathrm{GDPNP}$, and Thr61 from EF-Tu.GDPNP (1EXM). The distance between $\mathrm{N}$ of His84 and $\mathrm{O}$ of $\gamma$-phosphate is between $3 \AA$ and $5 \AA$ depending on the His 84 rotamer used. the switch II region, and the orientation of His84 must change in order to position the His84 sidechain in the catalytic site to stabilize the GTPase transition state. Intrinsic GTP hydrolysis in EF-Tu is slow, probably because the spontaneous structural rearrangement of His84 takes place rarely or not at all. In the latter case, the mechanism of GTP hydrolysis would take a pathway different from that supported by the ribosome, relying on different catalytic residues in the active center. The antibiotic aurodox induces a structure of EF-Tu with His84 oriented towards the GTP-binding pocket in a conformation that is unique in comparison to all other EF-Tu structures solved so far. ${ }^{10}$ In view of the crucial role of His84 for GTP hydrolysis, it appears likely that the structure of the active site in the EF-Tu-antibiotic complex is similar to that in the GTPase transition state induced on the ribosome. The fact that aurodox (or kirromycin) stimulated GTP hydrolysis only moderately (tenfold), measured under conditions of multiple turnover, compared to the extensive stimulation by the ribosome, can be explained by slow turnover. Thus, the antibiotic may induce the GTPase-activated conformation of EF-Tu, while it blocks subsequent structural rearrangements that determine the rate of turnover.

The ribosome stimulates the intrinsic GTP hydrolysis by EF-Tu in the ternary complex by five orders of magnitude. ${ }^{28}$ This can be achieved either by promoting the conformational rearrangements that correctly position the own catalytic groups of EF-Tu, i.e. His 84 or other residues at the active site, or by donating additional catalytic groups in trans. A residue next to His84, Gly83, plays an important role in both the rearrangement of the switch II region upon GTPase activation, due to the great flexibility inherent to Gly residues, and in GTP hydrolysis itself, probably by helping to position the catalytic water by hydrogen-bonding with the main-chain oxygen atom of Gly83 (Figure 6). ${ }^{18}$ The intrinsic arginine residue (Arg58), that is located in the switch I region of EF-Tu in a position homologous to that of the catalytic arginine in $G_{\alpha}$ proteins, is not essential for GTPase activity, ${ }^{29}$ and an extensive mutagenesis search did not identify any other side-chains important for GTP hydrolysis in E. coli EF-Tu. ${ }^{29,30-32}$ Thus, two residues in EF-Tu, Gly83 and His84, seem to provide the most important contributions to GTP hydrolysis.

The cryo-EM reconstruction of the ternary complex stalled on the ribosome after GTP hydrolysis, but prior to the conformational rearrangement into the GDP form, shows extensive interactions of the switch regions of the $G$ domain with the sarcin-ricin loop (SRL) of $23 \mathrm{~S}$ rRNA, ${ }^{33,34}$ indicating that the SRL stabilizes the transition state conformation of the switch regions of the factor. Another contact is formed between the elbow region of aa-tRNA in the ternary complex and ribosomal protein $\mathrm{L} 11^{33}$ or the L11-binding region of $23 \mathrm{~S}$ rRNA, ${ }^{34}$ and there is a transient contact of ribosomal protein L7/12 to helix D of EF-Tu ${ }^{35}$ (unpublished results). On the basis of biochemical and 
genetic analyses, the contacts with L7/12, L11, and the L11-binding region of $23 \mathrm{~S}$ rRNA are involved in stimulating GTP hydrolysis by EF-Tu on the ribosome. ${ }^{36,37}$ However, all these contacts take place far from the nucleotide-binding pocket and therefore must act indirectly by inducing conformational transitions of EF-Tu, rather than donate catalytic groups for GTP hydrolysis, as in the case of Ras. ${ }^{7}$

Finally, another key player in determining the rate of GTP hydrolysis appears to be aa-tRNA, which in the ternary complex contacts both switches I and II regions of the G domain of EF-Tu. The correct geometry of the codon-anticodon interaction on the $30 \mathrm{~S}$ subunit $^{22}$ and an intact tRNA molecule in the ternary complex ${ }^{38}$ are both required to induce rapid GTP hydrolysis. In the ternary complex stalled on the ribosome in the presence of kirromycin, the interactions between aa-tRNA and EF-Tu are distorted ${ }^{33,34}$ such that the contacts of the acceptor domain of tRNA with EF-Tu are likely to be altered and the switch regions of the factor may undergo a tRNA/ribosome-driven conformational change resulting in repositioning of His84. Thus, although the molecular details of GTPase activation and GTP hydrolysis remain to be determined directly, the combined influence of the ribosome and the tRNA appear to promote rearrangements of the $G$ domain of EF-Tu that position the catalytic residue(s), in particular the essential His84, into the active site such as to stabilize the reactive arrangement of the attacking water molecule or, possibly, the $\gamma$-phosphate of GTP.

\section{Materials and Methods}

\section{Buffer and reagents}

All experiments were performed in buffer A $(50 \mathrm{mM}$ Tris- $\mathrm{HCl}$ (pH 7.5), $50 \mathrm{mM} \mathrm{NH} \mathrm{NH}_{4} \mathrm{Cl} 10 \mathrm{mM} \mathrm{MgCl}_{2}$, $1 \mathrm{mM}$ DTT). The experiments were performed at $20{ }^{\circ} \mathrm{C}$, if not stated otherwise. Chemicals were purchased from Roche or Merck, radioactive compounds from ICN. mant-GTP and mant-GTPyS were from JenaBioScience.

\section{Ribosome complex}

Ribosomes from E.coli, AcPhe-tRNA ${ }^{\text {Phe }}$ from E.coli, $\left[{ }^{14} \mathrm{C}\right]$ Phe-tRNA ${ }^{\text {Phe }}$, and tRNA $^{\text {Phe }}($ Prf16/17) from yeast were prepared as described. ${ }^{17,39}$ To prepare $\mathrm{P}$ siteblocked ribosomes, $70 \mathrm{~S}$ ribosomes were incubated in buffer $\mathrm{A}$ for 15 minutes at $37^{\circ} \mathrm{C}$ with a 1.3-fold excess of AcPhe-tRNA $^{\text {Phe }}$ or tRNA $^{\text {Phe }}$ from E. coli and $0.5 \mathrm{mg} / \mathrm{ml}$ of $\operatorname{poly}(\mathrm{U})$.

\section{EF-Tu mutant}

Single-stranded DNA from the plasmid pEECAHis ${ }^{40}$ containing the E. coli tufA gene was used for site-directed mutagenesis with the Chameleon ${ }^{\mathrm{TM}}$ mutagenesis kit (Stratagene, Heidelberg), using the primer $5^{\prime}$ CATAGTC GGCGGCCCCCGGGCAGTCTACG3'. The mutation resulting in the H84A replacement was verified by DNA sequencing. Mutant EF-Tu(H84A) containing a C-terminal histidine tag was expressed in E. coli BL21 DE3 and purified by Ni-NTA-affinity chromatography. Cells were opened by sonication in buffer B $(50 \mathrm{mM}$ Tris- $\mathrm{HCl}(\mathrm{pH} 8.0), 60 \mathrm{mM} \mathrm{NH}{ }_{4} \mathrm{Cl}, 7 \mathrm{mM} \mathrm{MgCl}, 7 \mathrm{mM}$ 2-mercaptoethanol, $1 \mathrm{mM}$ PMSF, $50 \mu \mathrm{M}$ GDP, $300 \mathrm{mM}$ $\mathrm{KCl}, 10 \mathrm{mM}$ imidazole, $15 \%$ glycerol). After centrifugation for 30 minutes at 10,000 rpm (A8.24 rotor, Kontron), the supernatant was mixed with Ni-NTA Sepharose (Qiagen) equilibrated with buffer B and incubated for 30 minutes at $4{ }^{\circ} \mathrm{C}$. The supernatant was removed, and the Ni-NTA Sepharose was washed three times with 20 volumes of buffer B and an additional three times with 20 volumes of buffer B containing $20 \mathrm{mM}$ imidazole. Protein was eluted with $250 \mathrm{mM}$ imidazole in buffer $\mathrm{B}$ and subsequently rebuffered to buffer A using Centricon 30 concentrators (Amicon).

The concentration of EF-Tu was determined photometrically at $280 \mathrm{~nm}^{41}$ using a molar extinction coefficient of $32,900 \mathrm{M}^{-1} \mathrm{~cm}^{-1}$ and verified by comparing the intensity of the Coomassie-stained protein band on an SDS- $12 \%(\mathrm{w} / \mathrm{v})$ polyacrylamide gel with that of a standard protein using an imaging densitometer (Biorad GS-700).

\section{Ternary complex}

Ternary complex, EF-Tu(H84A).GTP.Phe-tRNA ${ }^{\text {Phe }}$, was prepared and purified by gel-filtration on Superdex 75 in buffer $\mathrm{A}$ as described ${ }^{17}$ with the following modifications. To prepare EF-Tu(H84A).GTP.Phe-tRNA ${ }^{\text {Phe }}(\operatorname{Prf16} / 17)$ complexes, $10 \mu \mathrm{M}$ tRNA ${ }^{\text {Phe }}(\operatorname{Prf16} / 17)$ were incubated with $30 \mu \mathrm{M}\left[{ }^{14} \mathrm{C}\right]$ Phe, $5 \%$ (v/v) S100 cell extract as a source of phenylalanyl-tRNA synthetase, $30 \mu \mathrm{M}$ EF-Tu, $1 \mathrm{mM}$ GTP, $3 \mathrm{mM}$ ATP, $3 \mathrm{mM}$ phosphoenol pyruvate, and $0.1 \mathrm{mg} / 1$ of pyruvate kinase in buffer $A$ for 30 minutes, $37^{\circ} \mathrm{C}$. EF-Tu(H84A).mant-GTP.Phe-tRNA ${ }^{\text {Phe }}$ complexes were prepared in the same way, except that $300 \mu \mathrm{M}$ mant-GTP was used instead of GTP. To prepare EF-Tu-mant-GTP $\gamma S$.Phe-tRNA ${ }^{\text {Phe }}, 17 \mu \mathrm{M}\left[{ }^{14} \mathrm{C}\right]$ PhetRNA $^{\text {Phe }}$ were incubated with $50 \mu \mathrm{M}$ EF-Tu and $200 \mu \mathrm{M}$ mant-GTP $\gamma \mathrm{S}$ in the presence of $0.02 \mu \mathrm{M}$ EF-Ts in buffer A for 30 minutes, $37^{\circ} \mathrm{C}$, and the ternary complex was purified by gel-filtration.

\section{Ribosome-EF-Tu-tRNA complex}

The $K_{d}$ of ternary complex binding to ribosomes was determined using non-purified ternary complex prepared with a tenfold excess of EF-Tu(H84A) over $\left[{ }^{14} \mathrm{C}\right]$ Phe-tRNA ${ }^{\text {Phe }}$. Ternary complex $(2 \mathrm{nM})$ was incubated with 0.1-50 nM P site-blocked ribosomes for one minute at $20^{\circ} \mathrm{C}$ and then passed through nitrocellulose filters (Sartorius $0.45 \mu \mathrm{m}$ ). Filters were washed with $5 \mathrm{ml}$ of buffer A, dissolved in scintillation fluid (QS361, Zinsser Analytic) and counted. For chase experiments, ternary complex and $\mathrm{P}$ site-blocked ribosomes were mixed to form a codon-recognition complex at a concentration of $0.6 \mu \mathrm{M}$. An equal volume of $6 \mu \mathrm{M}$ ternary complex with unlabeled Phe-tRNA ${ }^{\text {Phe }}$ was added, samples were taken after different incubation times and analyzed by nitrocellulose filtration as described above.

\section{Kinetic experiments}

Fluorescence stopped-flow measurements were performed as described previously ${ }^{17}$ using a stopped-flow 
apparatus from Applied Photophysics. Proflavin fluorescence was excited at $470 \mathrm{~nm}$ and measured after passing KV 500 filters (Schott). The fluorescence of mant-GTP was excited at $366 \mathrm{~nm}$ and measured after passing KV 408 filters (Schott). Experiments were performed by rapidly mixing equal volumes $(60 \mu \mathrm{l}$ each) of ternary complex $(0.2 \mu \mathrm{M})$, purified by gel filtration, and P site-blocked ribosomes $(0.6-3 \mu \mathrm{M})$ at $20^{\circ} \mathrm{C}$.

For the chase experiments, equal amounts of $\mathrm{P}$ site-blocked ribosomes and ternary complex, EF-Tu(H84A). GTP.Phe-tRNA ${ }^{\text {Phe }}(\operatorname{Prf16} / 17)$, were mixed to form $0.2 \mu \mathrm{M}$ codon-recognition complex. The dissociation of the complex was initiated in the stopped-flow apparatus by mixing with an equal volume of $2 \mu \mathrm{M}$ non-fluorescent ternary complex, EF-Tu(H84A)-GTP.Phe-tRNA ${ }^{\text {Phe }}$

Time-courses for GTP hydrolysis and dipeptide formation were measured manually under conditions identical with those employed in the stopped-flow experiments. Purified ternary complex, EF-Tu(H84A): $\left[\gamma^{32} \mathrm{P}\right] \mathrm{GTP} \cdot\left[{ }^{14} \mathrm{C}\right]$ Phe-tRNA ${ }^{\text {Phe }}$, and $\mathrm{P}$ site-blocked ribosomes were mixed to obtain final concentrations of $0.1 \mu \mathrm{M}$ and $0.3 \mu \mathrm{M}$, respectively, in a reaction volume of $800 \mu \mathrm{l}$. To measure GTP hydrolysis rates, samples were quenched with $25 \%(\mathrm{v} / \mathrm{v})$ formic acid and analyzed by thin-layer chromatography on polyethyleneimine-cellulose plates in $0.5 \mathrm{M}$ potassium phosphate, $\mathrm{pH} 3.5$, and quantified using a Biorad phosphoimager. To measure rates of dipeptide formation, samples were quenched with $0.6 \mathrm{M} \mathrm{KOH}$, incubated for 30 minutes at $37^{\circ} \mathrm{C}$, neutralized, and dipeptide formed was analyzed by HPLC.

The $\mathrm{pH}$ dependence of GTP hydrolysis was measured using ternary complex containing wild-type EF-Tu and mant-GTP $\gamma \mathrm{S}$ in the fluorescence stopped-flow apparatus. Prior to the experiment, ternary and ribosomal complex were diluted 1:10 into buffer A additionally containing $20 \mathrm{mM}$ Bis-Tris at $\mathrm{pH}$ 6.5-8.5. Equal volumes of ternary complexes and $\mathrm{P}$ site-blocked ribosomes, $0.1 \mu \mathrm{M}$ and $0.3 \mu \mathrm{M}$, respectively, in the buffer of the respective $\mathrm{pH}$ were rapidly mixed and the mantGTP $\gamma S$ fluorescence monitored.

To obtain values for apparent rate constants, timecourses were evaluated by fitting an expression which contained one or more exponential terms (characterized by variable time constants, $k_{\text {app }}$, and amplitudes) and another variable for the final signal using TableCurve software (Jandel Scientific) or GraphPad Prism ${ }^{\mathrm{TM}}$.

\section{Calculation of rate constants}

Values of $k_{1}$ and $k_{-1}$ were determined from the slope and $Y$-axis intercept, respectively, of the concentration dependence of $k_{\mathrm{app}}$ of initial binding measured in a model system where only this step was observed. ${ }^{19}$ For the calculation of $k_{2}$ and $k_{3}$, the concept of net rate constants was used..$^{25}$ The suitability of this approach for the analysis of data obtained under pre-steadystate conditions was shown earlier. ${ }^{42}$ Rate constants were calculated for the model depicted in Figure 5 From the concentration dependencies of the rates of codon recognition (step 2, $k_{\text {app } 2}$ ) and GTPase activation (step $\left.3, k_{\text {app } 3}\right)$, values for $k_{\text {app }}$ at saturation $\left(k_{\text {app2 } 2}\right.$ and $k_{\text {app3 }}$, respectively), and the concentration of ribosomes at half saturation, $K_{\mathrm{M} 2}$ and $K_{\mathrm{M} 3}$, respectively, were determined by hyperbolic fitting. Derivation of the concentration dependencies of the apparent rates $\left(k_{\text {app }}\right)$ of steps 2 and 3 yields: ${ }^{25}$

$k_{\mathrm{app} 2}=k_{\mathrm{sat} 2}[\mathrm{Rs}] /\left(K_{\mathrm{M} 2}+[\mathrm{Rs}]\right)$,

where $k_{\mathrm{sat} 2}=k_{2}$ and $K_{\mathrm{M} 2}=\left(k_{-1}+k_{2}\right) / k_{1}$,

$k_{\mathrm{app} 3}=k_{\mathrm{sat} 3}[\mathrm{Rs}] /\left(K_{\mathrm{M} 3}+[\mathrm{Rs}]\right)$,

where $k_{\text {sat3 }}=k_{2} k_{3} /\left(k_{2}+k_{3}\right)$

and $K_{\mathrm{M} 3}=\left(k_{-1}+k_{2}\right) / k_{1} k_{3} /\left(k_{2}+k_{3}\right)$

where [Rs] is the concentration of ribosomes. The value of $K_{\mathrm{d}}$ of steps $1-3$ was calculated as $\left(k_{-1} k_{-2} k_{-3}\right) /\left(k_{1} k_{2} k_{3}\right)$ from the rate constants of Figure 5 and compared with the value determined experimentally (Figure 3).

\section{Acknowledgements}

We thank Wolfgang Wintermeyer for valuable comments on the manuscript. We acknowledge the contributions of Yuri Semenkov and Maria Ludewig to the initial phase of this work. We also thank Vladimir Katunin and Yuri Semenkov for tRNA preparations, and Astrid Böhm, Petra Striebeck, Carmen Schillings and Simone Möbitz for expert technical assistance. The work was supported by the Deutsche Forschungsgemeinschaft, the Alfried Krupp von Bohlen und HalbachStiftung, and the Fonds der Chemischen Industrie. T.D. was supported by a fellowship of the Boehringer Ingelheim Fonds.

\section{References}

1. Pai, E. F., Krengel, U., Petsko, G. A., Goody, R. S., Kabsch, W. \& Wittinghofer, A. (1990). Refined crystal structure of the triphosphate conformation of H-ras p21 at $1.35 \AA$ resolution: implications for the mechanism of GTP hydrolysis. EMBO J. 9, 2351-2359.

2. Berchtold, H., Reshetnikova, L., Reiser, C. O., Schirmer, N. K., Sprinzl, M. \& Hilgenfeld, R. (1993). Crystal structure of active elongation factor $\mathrm{Tu}$ reveals major domain rearrangements. Nature, 365, 126-132.

3. Schweins, T., Langen, R. \& Warshel, A. (1994). Why have mutagenesis studies not located the general base in ras p21. Nature Struct. Biol. 1, 476-484.

4. Schweins, T. \& Warshel, A. (1996). Mechanistic analysis of the observed linear free energy relationships in p21ras and related systems. Biochemistry, 35, 14232-14243.

5. Schweins, T., Geyer, M., Scheffzek, K., Warshel, A., Kalbitzer, H. R. \& Wittinghofer, A. (1995). Substrateassisted catalysis as a mechanism for GTP hydrolysis of p21ras and other GTP-binding proteins. Nature Struct. Biol. 2, 36-44.

6. Schweins, T., Geyer, M., Kalbitzer, H. R., Wittinghofer, A. \& Warshel, A. (1996). Linear free energy relationships in the intrinsic and GTPase activating proteinstimulated guanosine $5^{\prime}$-triphosphate hydrolysis of p21ras. Biochemistry, 35, 14225-14231.

7. Vetter, I. R. \& Wittinghofer, A. (1999). Nucleoside 
triphosphate-binding proteins: different scaffolds to achieve phosphoryl transfer. Quart. Rev. Biophys. 32, $1-56$.

8. Bourne, H. R., Sanders, D. A. \& McCormick, F. (1991). The GTPase superfamily: conserved structure and molecular mechanism. Nature, 349, 117-127.

9. Hilgenfeld, R. (1995). Regulatory GTPases. Curr. Opin. Struct. Biol. 5, 810-817.

10. Vogeley, L., Palm, G. J., Mesters, J. R. \& Hilgenfeld, R. (2001). Conformational change of elongation factor $\mathrm{Tu}$ (EF-Tu) induced by antibiotic binding. Crystal structure of the complex between EF-Tu.GDP and aurodox. J. Biol. Chem. 276, 17149-17155.

11. Parmeggiani, A. \& Swart, G. W. (1985). Mechanism of action of kirromycin-like antibiotics. Annu. Rev. Microbiol. 39, 557-577.

12. Pape, T., Wintermeyer, W. \& Rodnina, M. V. (1998). Complete kinetic mechanism of elongation factor Tu-dependent binding of aminoacyl-tRNA to the A site of the E. coli ribosome. EMBO J. 17, 7490-7497.

13. Krab, I. M. \& Parmeggiani, A. (1999). Mutagenesis of three residues, isoleucine-60, threonine-61, and aspartic acid-80, implicated in the GTPase activity of Escherichia coli elongation factor Tu. Biochemistry, 38, 13035-13041.

14. Cool, R. H. \& Parmeggiani, A. (1991). Substitution of histidine-84 and the GTPase mechanism of elongation factor Tu. Biochemistry, 30, 362-366.

15. Scarano, G., Krab, I. M., Bocchini, V. \& Parmeggiani, A. (1995). Relevance of histidine-84 in the elongation factor Tu GTPase activity and in poly(Phe) synthesis: its substitution by glutamine and alanine. FEBS Letters, 365, 214-218.

16. Zeidler, W., Egle, C., Ribeiro, S., Wagner, A., Katunin, V., Kreutzer, R. et al. (1995). Site-directed mutagenesis of Thermus thermophilus elongation factor Tu. Replacement of His85, Asp81 and Arg300. Eur. J. Biochem. 229, 596-604.

17. Rodnina, M. V., Fricke, R. \& Wintermeyer, W. (1994). Transient conformational states of aminoacyl-tRNA during ribosome binding catalyzed by elongation factor Tu. Biochemistry, 33, 12267-12275.

18. Knudsen, C., Wieden, H. J. \& Rodnina, M. V. (2001). The importance of structural transitions of the switch II region for the functions of elongation factor $\mathrm{Tu}$ on the ribosome. J. Biol. Chem. 276, 22183-22190.

19. Rodnina, M. V., Pape, T., Fricke, R., Kuhn, L. \& Wintermeyer, W. (1996). Initial binding of the elongation factor Tu-GTP.aminoacyl-tRNA complex preceding codon recognition on the ribosome. J. Biol. Chem. 271, 646-652.

20. Rodnina, M. V., Fricke, R., Kuhn, L. \& Wintermeyer, W. (1995). Codon-dependent conformational change of elongation factor Tu preceding GTP hydrolysis on the ribosome. EMBO J. 14, 2613-2619.

21. Paulsen, H., Robertson, J. M. \& Wintermeyer, W. (1982). Effect of ribosome binding and translocation on the anticodon of RNA $^{\text {Phe }}$ as studied by wybutine fluorescence. Nucl. Acids Res. 10, 2651-2663.

22. Pape, T., Wintermeyer, W. \& Rodnina, M. V. (1999) Induced fit in initial selection and proofreading of aminoacyl-tRNA on the ribosome. EMBO J. 18, 3800-3807.

23. Thompson, R. C. (1988). EFTu provides an internal kinetic standard for translational accuracy. Trends Biochem. Sci. 13, 91-93.

24. Gromadski, K. B., Wieden, H. J. \& Rodnina, M. V. (2002). Kinetic mechanism of elongation factor Ts- catalyzed nucleotide exchange in elongation factor Tu. Biochemistry, 41, 162-169.

25. Cleland, W. W. (1975). Partition analysis and the concept of net rate constants as tools in enzyme kinetics. Biochemistry, 14, 3220-3224.

26. Karim, A. M. \& Thompson, R. C. (1986). Guanosine 5'-O-(3-thiotriphosphate) as an analog of GTP in protein biosynthesis. The effects of temperature and polycations on the accuracy of initial recognition of aminoacyl-tRNA ternary complexes by ribosomes. J. Biol. Chem. 261, 3238-3243.

27. Helms, M. K., Marriott, G., Sawyer, W. H. \& Jameson, D. M. (1996). Dynamics and morphology of the in vitro polymeric form of elongation factor $\mathrm{Tu}$ from Escherichia coli. Biochim. Biophys. Acta, 1291, 122-130.

28. Rodnina, M. V., Pape, T., Fricke, R. \& Wintermeyer, W. (1995). Elongation factor Tu, a GTPase triggered by codon recognition on the ribosome: mechanism and GTP consumption. Biochem. Cell Biol. 73, 1221-1227.

29. Knudsen, C. R. \& Clark, B. F. (1995). Site-directed mutagenesis of Arg58 and Asp86 of elongation factor $\mathrm{Tu}$ from Escherichia coli: effects on the GTPase reaction and aminoacyl-tRNA binding. Protein Eng. 8, 1267-1273.

30. Mansilla, F., Knudsen, C. R., Laurberg, M. \& Clark, B. F. (1997). Mutational analysis of Escherichia coli elongation factor $\mathrm{Tu}$ in search of a role for the N-terminal region. Protein Eng. 10, 927-934.

31. Rattenborg, T., Nautrup Pedersen, G., Clark, B. F. \& Knudsen, C. R. (1997). Contribution of Arg288 of Escherichia coli elongation factor $\mathrm{Tu}$ to translational functionality. Eur. J. Biochem. 249, 408-414.

32. Wiborg, O., Andersen, C., Knudsen, C. R., Clark, B. F. \& Nyborg, J. (1996). Mapping Escherichia coli elongation factor $\mathrm{Tu}$ residues involved in binding of aminoacyl-tRNA. J. Biol. Chem. 271, 20406-20411.

33. Stark, H., Rodnina, M. V., Wieden, H.-J., Zemlin, F., Wintermeyer, W. \& van Heel, M. (2002). Ribosome interactions of aminoacyl-tRNA and elongation factor $\mathrm{Tu}$ in the codon recognition complex. Nature Struct. Biol. 9, 849-854.

34. Valle, M., Sengupta, J., Swami, N. K., Grassucci, R. A., Burkhardt, N., Nierhaus, K. H., Agrawal, R. K. \& Frank, J. (2002). Cryo-EM reveals an active role for aminoacyl-tRNA in the accommodation process. EMBO J. 21, 3557-3567.

35. Stark, H., Rodnina, M. V., Rinke-Appel, J., Brimacombe, R., Wintermeyer, W. \& van Heel, M. (1997). Visualization of elongation factor Tu on the Escherichia coli ribosome. Nature, 389, 403-406.

36. Rodnina, M. V., Pape, T., Savelsbergh, A., Mohr, D., Matassova, N. B. \& Wintermeyer, W. (2000). Mechanisms of partial reactions of the elongation cycle catalyzed by elongation factors $\mathrm{Tu}$ and $\mathrm{G}$; In The Ribosome: Structure, Functiom, Antibiotics, and Cellular Interactions (Garrett, R. A., Douthwaite, S. R., Liljas, A., Matheson, A. T., Moore, P. B. \& Noller, H. F., eds), pp. 301-317, ASM Press, Washington, DC.

37. Mohr, D., Wintermeyer, W. \& Rodnina, M. V. (2002). GTPase activation of elongation factors $\mathrm{Tu}$ and $\mathrm{G}$ on the ribosome. Biochemistry, 41, 12520-12528.

38. Piepenburg, O., Pape, T., Pleiss, J. A., Wintermeyer, W., Uhlenbeck, O. \& Rodnina, M. V. (2000). Intact aminoacyl-tRNA is required to trigger GTP hydrolysis by elongation factor Tu on the ribosome. Biochemistry, 39, 1734-1738. 
39. Rodnina, M. V. \& Wintermeyer, W. (1995). GTP consumption of elongation factor Tu during translation of heteropolymeric mRNAs. Proc. Natl Acad. Sci. USA, 92, 1945-1949.

40. Wieden, H. J., Gromadski, K., Rodnin, D. \& Rodnina, M. V. (2002). Mechanism of elongation factor (EF)-Tscatalyzed nucleotide exchange in EF-Tu. Contribution of contacts at the guanine base. J. Biol. Chem. 277, 6032-6036.
41. Block, W. \& Pingoud, A. (1981). The identification and analysis of nucleotides bound to the elongation factor Tu from Escherichia coli. Anal. Biochem. 114, 112-117.

42. Bilgin, N., Claesens, F., Pahverk, H. \& Ehrenberg, M. (1992). Kinetic properties of Escherichia coli ribosomes with altered forms of S12. J. Mol. Biol. 224, 1011-1027.

Edited by J. Doudna

(Received 27 May 2003; received in revised form 16 July 2003; accepted 17 July 2003) 